\title{
Criptosporidiosis en Caninos Criados en Comunidades Campesinas de Puno, Perú
}

\author{
Cryptosporidiosis in Dogs Bred in Rural Communities of Puno, Peru
}

Noemit Celis S. ${ }^{1}$, Amanda Chávez V. ${ }^{1,5}$, Francisco Suárez A. ${ }^{2}$, Néstor Falcón P. ${ }^{4}$, Viviana Fernández $\mathbf{P}^{3}$

\section{Resumen}

El objetivo del estudio fue determinar la prevalencia de Cryptosporidium sp en caninos de comunidades campesinas de los distritos de Ajoyani, provincia de Carabaya, y de Palca y Santa Lucia, provincia de Lampa, en Puno, Perú. Se recolectaron 123 muestras fecales de canes aparentemente sanos, de ambos sexos y con edades mayores de un mes, y se analizaron mediante la técnica de Ziehl-Neelsen modificada para el diagnóstico de Cryptosporidium sp. La prevalencia general de Cryptosporidium sp fue de $26.8 \pm$ $7.8 \%$, sin encontrarse asociación significativa entre la presencia del protozoo con el distrito de procedencia, sexo y edad. Los resultados demuestran la presencia de Cryptosporidium sp en caninos evidenciando un posible riesgo zoonótico.

Palabras clave: Cryptosporidium sp, protozoo, zoonosis, prevalencia, perros, ZiehlNeelsen modificada

\section{Abstract}

The aim of this study was to determine the prevalence of Cryptosporidium sp in dogs of rural communities located in the districts of Ajoyani, Carabaya province and Palca and Santa Lucia, Lampa province, in Puno, Peru. Fecal samples were collected $(n=123)$ from apparently healthy dogs of both sexes, older than one month. The ZiehlNeelsen modified technique was used for the diagnosis of Cryptosporidium sp. The

\footnotetext{
${ }^{1}$ Laboratorio de Parasitología y Microbiología Veterinaria, ${ }^{2}$ Laboratorio de Medicina Veterinaria Preventiva, ${ }^{3}$ Clínica de Animales Menores, Facultad de Medicina Veterinaria, Universidad Nacional Mayor de San Marcos, Lima, Perú

${ }^{4}$ Facultad de Medicina Veterinaria y Zootecnia, Universidad Peruana Cayetano Heredia, Lima, Perú

${ }^{5}$ E-mail: achavezvg@gmail.com
}

Recibido: 25 de febrero de 2014

Aceptado para publicación: 20 de diciembre de 2014 
overall prevalence was $26.8 \pm 7.8 \%$ for Cryptosporidium sp and without significant association between the presence of the protozoa with the district, sex and age. The results show the presence of Cryptosporidium $\mathrm{sp}$ in dogs evidencing a possible zoonotic risk.

Key words: Cryptosporidium sp, protozoan, zoonotic, prevalence, dogs, Ziehl-Neelsen modified

\section{INTRODUCCIÓN}

La criptosporidiosis es una enfermedad parasitaria zoonótica de distribución cosmopolita (Ortega et al., 1999), producida por protozoos del género Cryptosporidium $\mathrm{sp}$ que afectan a una gran variedad de reptiles, peces, aves y mamíferos, incluyendo al humano y al canino (Acha y Szyfres, 2003).

Actualmente, Cryptosporidium parvum es la especie de mayor interés dentro del género, dado que se multiplica preferentemente en las células epiteliales del intestino delgado de los mamíferos (Ortega et al., 1999), desencadenando un proceso diarreico agudo autolimitado en individuos inmunocompetentes, con una duración aproximada de dos semanas y una patología crónica en pacientes inmunodeprimidos (SIDA/VIH, cáncer, quimioterapia antineoplásica, malnutrición, etc.), pudiendo persistir hasta la muerte del hospedador (Huiza et al., 2004). Debido a su escasa especificidad, puede transmitirse al humano, mamíferos diversos y ocasionalmente a gallinas (Urquhart et al., 2001).

En todas las especies domésticas, los animales que se encuentran en lactación son los más susceptibles a la infección (Acha y Szyfres, 2003). En los niños, Cryptosporidium $\mathrm{sp}$ es la tercera causa de diarrea infecciosa, generalmente después de los rotavirus y de Escherichia coli (De Arango et al., 2006).

Los animales de compañía, en especial canes y gatos, contribuyen con el desenvol- vimiento físico, social y emocional de las personas, tanto en los niños como en sus propietarios, en particular los ancianos; sin embargo, estos animales pueden constituir una importante fuente de infección para el hombre, determinando enfermedades zoonóticas, como el caso de la criptosporidiosis (Greene, 2000; Vergara y Quílez, 2004). Personas carentes de cuidados sanitarios e higiene personal, así como inmunodeprimidos pueden adquirir infecciones transmitidas por sus mascotas (Dabanch, 2003; Shukla et al., 2006).

En Lima Metropolitana se han realizado escasos estudios sobre C. parvum en animales de compañía. Se dispone de un reporte en gatos de diversas edades con $6.9 \%$ de infección (Rojas, 2004), mientras que Romero et al. (2000) y Sotelo et al. (2013) reportaron una prevalencia de 25.4 y $29.7 \%$ en caninos, respectivamente. Por otro lado, en un estudio en 450 caninos de la ciudad de São Paulo, se reportó una prevalencia de $8.8 \%$ para Cryptosporidium sp (Lallo y Bondan, 2006); en tanto que en la ciudad de Tunja, Colombia, se encontró $16.4 \%$ de casos positivos al analizar 132 muestras de heces de perros de tres consultorios veterinarios (Rodríguez et al., 2009).

El primer reporte sobre la transmisión de la criptosporidiosis (C. canis) entre humanos y perros se hizo en un estudio de cohorte longitudinal con dos hermanos y su perro, donde los hermanos presentaron una diarrea transitoria y el perro se encontraba asintomático (Xiao et al., 2007). 
Considerando la importancia de la criptosporidiosis desde el punto de vista de salud pública debido a las diferentes causas que favorecen su presentación en el hombre, entre ellas la estrecha relación entre niños y perros donde el canino desempeña un papel epidemiológico importante, se diseñó el presente estudio con el fin de determinar la prevalencia de Cryptosporidium $\mathrm{sp}$ en caninos de comunidades campesinas de las provincia de Carabaya y Lampa, Puno.

\section{Materiales y Métodos}

\section{Lugar de Estudio}

El departamento de Puno se localiza en la parte sur oriental del Perú y está conformado por 13 provincias y 104 distritos. Presenta un clima frío y semiseco, con dos estaciones climáticas marcadas, una lluviosa y templada de diciembre a marzo y otra seca e invernal de abril a noviembre (Cuba e Ita, 2008; INEI, 2010).

El estudio se realizó en 2008 en los distritos de Ajoyani, provincia de Carabaya, y de Palca y Santa Lucía, provincia de Lampa, todos en el departamento de Puno, Perú, en altitudes superiores a los $4000 \mathrm{msnm}$. El muestreo se realizó entre enero y marzo (época de lluvias) de 2008 con temperaturas que fluctuaron entre -5 y $18.8^{\circ} \mathrm{C}$ y con precipitaciones de 187, 119 y $86 \mathrm{~mm}$ para Ajoyani, Palca y de Santa Lucía, respectivamente (SENAMHI, 2008).

\section{Charlas Informativas}

Se realizaron charlas informativas en los Centros de Salud de cada localidad para motivar la colaboración de la comunidad en la toma de muestras, así como para informar sobre las posibles enfermedades zoonóticas que pueden ser transmitidas por los caninos. Asimismo, se distribuyeron envases de plástico para las muestras de heces a los propietarios de canes que estuvieron dispuestos a participar en el estudio.

\section{Toma de Muestras}

Se trabajó con muestras fecales de caninos mestizos, de edad variada y de ambos sexos. Las heces recolectadas estaban libres de tierra y orina. Se recolectó una sola muestra por perro indicándose la procedencia, edad y sexo. Las muestras fueron trasportadas en cajas térmicas con geles refrigerantes y llevadas al Laboratorio de Parasitología de la estación experimental de Quimsachata del Instituto Nacional de Innovación Agraria (INIA), Puno. Se realizaron los frotices fecales, que luego se fijaron en metanol durante 5 min en vaso koplin. Las láminas resultantes se llevaron al Laboratorio de Parasitología de la Facultad de Medicina Veterinaria, Universidad Nacional Mayor de San Marcos, Lima, para su procesamiento y evaluación.

Las muestras fueron evaluadas mediante la técnica de tinción para organismos ácido-resistentes Ziehl-Neelsen modificada (Rojas, 2004). Los reactivos se prepararon según protocolos establecidos (Ministerio de Salud, 1999; Romero et al., 2000). Se consideró como muestra positiva toda lámina que presentaba al menos un ooquiste con las características propias de Cryptosporidium $\mathrm{sp}$ (Barriga, 2002).

\section{Diseño Experimental}

El estudio fue descriptivo de corte transversal. El tamaño mínimo de muestras se determinó utilizando la fórmula para estimar una proporción (Daniel, 1996). Se utilizó una prevalencia de $8.8 \%$ determinada en perros de São Paulo, Brasil (Lallo y Bondan, 2006). En base a esto, se estimó un tamaño de 123 muestras fecales para caninos de propietarios residentes en los distritos bajo estudio. Las muestras por distritos se obtuvieron utilizando la fórmula de estratificación (Pérez, 2000).

\section{Análisis Estadístico}

Las variables de carácter cualitativo fueron agrupadas en categorías para su aná- 
Cuadro 1. Prevalencia de Cryptosporidium sp en caninos según sexo y edad y localidad en muestras de las provincias de Carabaya y Lampa, Puno, analizadas mediante la técnica de Ziehl-Neelsen modificada (enero-marzo, 2008)

\begin{tabular}{|c|c|c|c|c|}
\hline \multirow{2}{*}{ Variables } & & \multirow{2}{*}{$\mathrm{N}^{\mathrm{o}}$ muestras } & \multicolumn{2}{|c|}{ Positivos } \\
\hline & & & N. ${ }^{o}$ & $\% \pm \mathrm{IC}^{1}$ \\
\hline \multirow{3}{*}{ Distritos } & Ajoyani & 21 & 4 & 19.0 \\
\hline & Palca & 21 & 6 & 28.6 \\
\hline & Santa Lucía & 81 & 23 & 28.4 \\
\hline \multirow{2}{*}{ Sexo } & Macho & 106 & 30 & 28.3 \\
\hline & Hembra & 17 & 3 & 17.6 \\
\hline \multirow{4}{*}{$\begin{array}{l}\text { Edad (en } \\
\text { meses) }\end{array}$} & 0 a 6 & 13 & 6 & 46.2 \\
\hline & $>6$ a 12 & 32 & 9 & 31.3 \\
\hline & $>12$ a 72 & 61 & 12 & 19.7 \\
\hline & $>72$ & 17 & 5 & 29.4 \\
\hline Total & & 123 & 33 & $26.8 \pm 7.8$ \\
\hline
\end{tabular}

lisis. Se evaluó el sexo (macho, hembra), edad $(0$ a $6,>6$ a $12,>12$ a $72,>72$ meses) y lugar de procedencia de la muestra (Ajoyani, Palca, Santa Lucía). La frecuencia de parasitosis se expresó en forma porcentual con sus respectivos intervalos de confianza del $95 \%$. Las posibles asociaciones de Cryptosporidium sp con edad, sexo y localidad se analizaron con la prueba de Chi cuadrado, utilizando el paquete estadístico SPSS v. 10.0 para Windows.

\section{Resultados}

La prevalencia general de Cryptosporidium sp fue de $26.8 \pm 7.8 \%$, sin encontrar asociación significativa entre las variables distrito, sexo o grupo etario con la presencia del parásito (Cuadro 1).

\section{Discusión}

Los caninos juegan un importante rol en la sociedad a nivel mundial, constituyendo una compañía importante en muchos hogares, apoyando al desarrollo emocional de niños y ancianos. Asimismo, en las comunidades rurales del Perú apoyan en las labores de pastoreo, donde mantienen un contacto directo con los niños. A pesar de los beneficios sociales y laborales de los perros, la prevalencia de la criptosporidiosis es un factor a considerar (Robertson et al., 2000; Acha y Szyfres, 2003), en especial en zona rurales donde existen otros factores de riesgo como bajo nivel socioeconómico, dificultad en la disponibilidad de agua, condiciones sanitarias deficientes, hacinamiento y escaso conocimiento de las medidas de higiene básicas (Botero y Restrepo, 2006). 
En el presente estudio se determinó una prevalencia general para Cryptosporidium sp de $26.8 \pm 7.8 \%$ en la zona en estudio. Lamentablemente, no existen estudios similares en caninos provenientes de otras zonas rurales en el país y solo se cuenta con los hallazgos obtenidos en zonas urbanas en Lima Metropolitana (Romero et al., 2000).

Los resultados hallados en este estudio difieren considerablemente con otros realizados en países vecinos. Así, Gorman et al. (2006) evaluaron caninos de clínicas veterinarias y los de Servicios de Higiene Ambiental en Chile encontrando una prevalencia de $1.9 \%$ para Cryptosporidium sp, mientras que Lallo y Bondan (2006) hallaron una prevalencia de $8.8 \%$ en caninos de un hospital veterinario universitario público y de perreras particulares en la cuidad de São Paulo. Por otro lado, caninos alojados en refugios después de su captura en el área metropolitana de Barcelona presentaron una prevalencia de 6.3\% (Gracenea et al., 2009); utilizándose la técnica de Ziehl-Neelsen modificada en todos los casos.

La similitud de resultados entre los tres distritos pudo deberse a las similares condiciones epidemiológicas de las zonas en estudio, donde la temperatura y precipitación en la estación de lluvias son ideales para la supervivencia del parásito en el medio ambiente. Por otro lado, los meses de muestreo coincidieron con la época de parición de las alpacas. Según Molina et al. (2009), el 34\% de las crías estarían parasitados en estas localidades; produciéndose así una contaminación progresiva en la explotación, favoreciendo la acumulación de materia fecal contaminada (Ortega et al., 1999; Quilez et al., 2003). Los perros pastores se encontrarían, de esta forma, en contacto directo con la materia fecal de los camélidos, teniendo una mayor probabilidad de infectarse.

Los canes de ambos sexos tienen las mismas oportunidades de infección (Ortega et al., 1999), de allí que no se encontrara una asociación significativa por efecto del sexo.
Similares hallazgos fueron reportados en caninos de ciudades urbanas (Romero et al., 2000; Lallo y Bondan, 2006; Rodríguez et al., 2009). Tampoco se encontró diferencia estadística entre la presentación de Cryptosporidium sp y la edad de los caninos, tal como fuera reportada por Romero et al. $(2000) \mathrm{y}$ Rodríguez et al. (2009); sin embargo, otros estudios indican que los individuos jóvenes son más susceptibles de contagio (Ortega et al., 1999; Acha y Szyfres, 2003).

Los resultados del presente estudio deberían alertar a las autoridades correspondientes sobre la importancia de la criptosporidiosis como enfermedad potencialmente zoonótica presente en la población, la cual está directamente relacionada con las medidas de higiene básicas (lavado de manos y alimentos, así como hervir el agua para consumo, etc.) y el saneamiento ambiental.

\section{Conclusiones}

- La prevalencia general de Cryptosporidium sp en caninos fue de $26.8 \pm$ $7.8 \%$ en los distritos de Ajoyani, provincia de Carabaya, y en los distritos de Palca y Santa Lucia, provincia de Lampa, departamento de Puno, Perú.

- No se encontró asociación significativa entre la presencia de Cryptosporidium sp con las variables distrito de procedencia, sexo y edad de los caninos.

- La presencia de Cryptosporidium sp en caninos evidencia un riesgo zoonótico en la población.

\section{Literatura Citada}

1. Acha PN, Szyfres B. 2003. Zoonosis y enfermedades transmisibles comunes al hombre y a los animales. $3^{\text {a }}$ ed. Washington: OPS. 398 p.

2. Barriga O. 2002. Las enfermedades parasitarias de los animales domésticos en la América Latina. Santiago de Chile: Germinal. 247 p. 
3. Botero D, Restrepo M. 2006. Parasitosis humanas. $4^{\text {a }}$ ed. Colombia: CBI. 506 p.

4. Cuba F, Ita N. 2008. Guía climática turística. Lima: SENAMHI. 216 p.

5. Dabanch PJ. 2003. Zoonosis. Rev Chil Infectol 20 (Supl 1): 47-51.

6. Daniel D. 1996. Bioestadística: base para el análisis de las ciencias de la salud. $5^{\text {a }}$ ed. México: Limusa. 878 p.

7. De Arango M, Rodríguez DA, Prada NE. 2006. Frecuencia de Cryptosporidium spp en materia fecal de niños entre un mes y trece años en un hospital local colombiano. Colomb Med 37: 121125.

8. Gorman T, Soto A, Alcaino H. 2006. Parasitismo gastrointestinal en perros de comunas de Santiago de diferente nivel socioeconómico. Parasitol Latinoam 61: 126-132.

9. Gracenea M, Gómez MS, Torres J. 2009. Prevalence of intestinal parasites in shelter dogs and cats in the metropolitan area of Barcelona (Spain). Acta Parasitol 54: 73-77. doi: 10.2478/ s11686-009-0005-7

10. Greene CE. 2000. Personas y mascotas inmunocomprometidas. En: Greene CE (ed). Enfermedades infecciosas en perros y gatos. $2^{\mathrm{a}}$ ed. México: McGrawHill Interamericana. p 784-791.

11. Huiza A, Espinoza Y, Rojas R, Sevilla C, Alva P, Verástegui R, Quispe E, et al. 2004. Detección de coccidios en niños asintomáticos mediante esporulación de muestras fecales. An Fac Med 65: 239-242.

12. [INEI] Instituto Nacional de Estadística e Informática. 2010. Lima: Presidencia de Consejo de Ministros. [Internet], [28 julio 2010]. Disponible en: http://www.inei.gob.pe/

13. Lallo MA, Bondan EF. 2006. Prevalência de Cryptosporidium sp em cães de instituições da cidade de São Paulo. Rev Saúde Públ 40: 120-125. doi: 10.1590/S0034-89102006000100019
14. Ministerio de Salud. 1999. Procedimientos de laboratorio. INS. Perú: Amarilys. 474 p.

15. Molina D, López T, González A, Gómez L, Pezo D. 2009. Cryptosporidium parvum como factor de riesgo en la diarrea neonatal en alpacas de Puno. Rev Inv Vet Perú 20: 263-269. doi: 10.15381/rivep.v20i2.620

16. Ortega ML, Gómez M, Rojo FA. 1999. Criptosporidiosis. En: Cordero del Campillo M, Rojo FA, Martínez AR, Sánchez MC, Hernández S, et al. (eds). Parasitología veterinaria. Madrid: McGraw-Hill Interamericana. p 213-221.

17. Pérez C. 2000. Técnicas de muestreo estadístico. 2000. México: Alfaomega. $603 \mathrm{p}$.

18. Quílez J, Sánchez-Acedo C, Del Cacho E. 2003. Criptosporidiosis de los pequeños rumiantes. Soc Española Ovinotecnia Caprinotecnia 4(2): 22-30.

19. Robertson ID, Irwin PJ, Lymbery AJ, Thompson RCA. 2000. The role of companion animals in the emergence of parasitic zoonoses. Int $\mathrm{J}$ Parasitol 30: 1369-1377. doi: 10.1016/S00207519(00)00134-X

20. Rodríguez, E, Manrique-Abril F, Pulido M, Ospina-Díaz J. 2009. Frecuencia de Cryptosporidium spp en caninos de la ciudad de Tunja-Colombia. Rev MVZ Córdoba 14: 1697-1704.

21. Rojas M. 2004. Nosoparasitismo de los rumiantes domésticos peruanos. $2^{\mathrm{a}}$ ed. Lima: UNMSM. 146 p.

22. Romero M, Chávez A, Casas E. 2000. Determinación de la presencia de Cryptosporidium parvum y Cyclospora sp. en caninos domésticos (Canis familiaris) en los distritos de Lima Metropolitana. Rev Inv Vet Perú 11: 26-31. doi: 10.15381/rivep.v11i1.6778

23. [SENAMHI] Servicio Nacional de Meteorología e Hidrología. 2008. Lima: Ministerio del Ambiente. [Internet], [08 abril 2010]. Disponible en: http://www. senamhi.gob.pe/ 
24. Sotelo H, Chávez A, Casas E, Pinedo R, Falcón N. 2013. Giardiasis y criptosporidiasis en caninos de los distritos del cono oeste de Lima Metropolitana. Rev Inv Vet Perú 24: 353-359. doi: 10.15381/rivep.v24i3.2584

25. Shukla R, Giraldo P, Kraliz A, Finnigan M, Sánchez A. 2006. Cryptosporidium spp and other zoonotic enteric parasites in a sample of domestic dogs and cats in the Niagara region of Ontario. Can Vet J 47: 1179-1184.
26. Urquhart GM, Armour J, Duncan JL, Dunn AM, Jenmngs FW. 2001. Parasitología veterinaria. $2^{\mathrm{a}}$ ed. Zaragoza: Acriba. $355 \mathrm{p}$.

27. Vergara C, Quílez, J. 2004. Criptosporidiosis: una zoonosis parasitaria. MVZ-Córdoba 9(1): 363-372.

28. Xiao L, Cama VA, Cabrera L, Ortega Y, Pearson J, Gilman RH. 2007. Possible transmission of Cryptosporidium canis among children and a dog in a household. J Clin Microbiol 45: 2014-2016. doi: 10.1128/JCM.00503-07 